\title{
Justification of the technical requirements of a fully functional modular robot
}

\author{
Nikita Shlyakhov ${ }^{1,2}$, Vladimir Dashevskiy ${ }^{1}$, Irina Vatamaniuk $^{1}$, Milos Zelezny ${ }^{3}$ and Andrey Ronzhin ${ }^{1,2^{*}}$ \\ ${ }^{1}$ SPIIRAS, Laboratory of Autonomous Robotic Systems, 199178 Saint-Petersburg, 14-th line of V.I., 39, Russia \\ ${ }^{2}$ SUAI, Department of Electromechanics and Robotics, 190000 Saint-Petersburg, Bolshaya Morskaya str., 67, Russia \\ ${ }^{3}$ University of West Bohemia, Pilsen, Czech Republic
}

\begin{abstract}
Modular robots are characterized by limited built-in resources necessary for communication, connection and movement of modules, when performing reconfiguration tasks at rigidly interconnected elements. In developing the technological fundamentals of designing modular robots with pairwise connection mechanisms, we analysed modern hardware and model algorithms typical of a fully functional robot, which provide independent locomotion, communication, navigation, decentralized power and control. A survey of actuators, batteries, sensors, communication means, suitable for modular robotics is presented.
\end{abstract}

\section{Introduction}

The complexity of developing robot group interaction algorithms in modular swarm robotics is related to the contact connection of homogeneous robotic elements to single structures in a three-dimensional space, limited by the capabilities of individual robots, due to small overall dimensions, low energy reserves, design features of propellers, etc. When reconfiguring the swarm of modular robots, algorithms for controlling robotic pairing of robots are necessary, both during the formation of the three-dimensional structure and after completion to maintain the shape of the swarm of the required configuration.

The most important specificity of modular robotics (MR) is the solution of kinematics problems of rigidly interconnected elements under conditions of limited built-in resources necessary for communication, connection and movement of modules, when performing reconfiguration tasks, assembling, capturing, moving a set of modular robots. In work [1], several basic tasks solved by MR are distinguished: reorganization on a surface or into a given form; movement; manipulations with external objects; support and balancing of unstable objects.

In self-reconfiguration, problems arise not only in the limited resources of individual modules, but also in collisions in the overall structure, for example: the separation of a modular robot into several parts; the overlap of individual modules in one position when planning a rebuild; formation of hollow closed forms, where a number of the modules remain inside and cannot be used further; and vice versa, when some of the modules remain outside and are not available to form an internal structure.
In work [2], a number of architectures of modular robots are considered depending on their structural parameters. The simplest variant is a group interaction of traditional robots with wheel/caterpillar propulsors, which are interconnected to increase their parameters when solving a particular problem. For robots whose whole body participates in movement and connection, there are three types of architecture: serpentine-like, lattice, hybrid. In this case, individual modules can take the form of a cube, a cylinder, a parallelepiped, a parallelepiped with rounded corners, trapezoidal, triangular, hexagonal, spherical and others.

For a modular robot consisting of homogeneous blocks with identical functional and physical characteristics, it is somewhat easier (from a computational point of view) to perform selfreconfiguration and solve other tasks. However, the use of universal blocks increases the cost of a modular robot, therefore, in a number of works it is proposed to use heterogeneous blocks, including passive elements. In many modular robots two types of main and connecting blocks are used [3]. In [4], 18 types of blocks are used, differing in form, connection mechanisms, methods of motion, capture, the presence of sensors and signal processing facilities.

When developing the mechanism for connecting blocks, one should take into account its necessary strength, reversibility and repeatability, power consumption, permissible geometric dimensions, as well as environmental conditions of operation. The mechanical properties of modular couplings impose certain limitations on the space of possible reconfigurations of the modular robot. In the majority of works mechanical, magnetic and chemical methods of connection are used, methods of connection based on artificial muscles are also actively investigated [5].

\footnotetext{
* Corresponding author: ronzhin@iias.spb.su
} 
A separate important issue in modular robotics is the communication problem, which in most cases is solved with the use of infra-red transceivers or wired communication means in the event of a permanent physical connection of all robot modules. Now the most widely used wireless communication tools are based on Bluetooth, ZigBee and other standards of the IEEE 802.15 protocol, as well as IEEE $802.11 \mathrm{WiFi}$. However, with wireless communication, as the number of modules increases, the problem of signal interference and channel capacity arises, given the small size and limited resources of the modules.

\section{Related works}

In [6], the motion of a closed chain-like modular robot in the rolling style is simulated. The complexity of the problem consists in calculating the position of each block, controlling the effects of the connecting mechanisms between two neighbouring blocks, which are necessary for the formation of motion, including on an uneven surface, and for maintaining the stable position of the entire modular robot. The experiments were carried out with a robot UBot during the selfrolling of a closed circuit in the form of a triangle and an oval along diverse types of surfaces, including the movement of the payload inside the chain.

The work [7] describes a novel approach to planning the reconfiguration of a modular robot based on the graph theory. The computational complexity of generating a graph structure is the logarithm of the number of modular robots involved. Calculations were made for modular robots M-TRAN and SuperBot with the number of involved elements up to 12 pieces.

The work [8] is dedicated to the problem of communication between modular robots which a priori do not have information about their situation and previous communication sessions. In the proposed approach of building a communication network using an infrared channel, neighbouring nearby modules are first identified, and then other robots are identified based on the XBee channel. The experiments were carried out with a maximum removal of 10 ModRED modular robots in an enclosed space of up to 30 meters and in an open space of up to 90 meters.

The review [9] presents the main tasks of rover robotics, behavioural algorithms (clustering, gathering in a swarm, collective food search), algorithms for clustering and sorting swarm elements, applied methods of navigation and communication, ways to distribute tasks within a robot swarm, as well as promising applications, solved on the basis of methods of swarm robotics.

In the work [10], the classification of modular robots is given and trellised and chain-like robots with 1D-3D topology are distinguished. Linear robots can be serpentine-like and serpenoid-like. The latter can move not only due to changes in shape but also built-in propellers (caterpillars, wheels, etc., installed on the outer surface of each block). For serpentine-like robots, three variants of pairwise connections and changes in the configuration of neighboring blocks are described: 1) in the vertical plane, which ensures the movement of the robot-snake only back and forth; 2) in the horizontal plane, which ensures movement in any direction along the plane; 3) in the vertical and horizontal planes, which ensures the movement of the robot-snake in threedimensional space. The latter option has a more complex mechanism, consisting of two elements that ensure the rotation of the neighboring block, respectively, in the vertical and horizontal planes. Also, the work considers 12 principles of serpentine movement, implemented on the basis of the proposed mathematical models and developed mechanisms of the modular robot-snake.

Thus, despite the new tasks of robotics in the field of medicine and transport, the tasks of modular robotics are still far from the final solution and are actively explored. The proposed technological bases for designing modular robots with pairwise coupling mechanisms describe modern hardware and model-algorithmic tools necessary for the development of promising models of modular robots $[11,12]$. These robots are equipped with systems typical of a fully functional robot, which provides their locomotion, communication, navigation, decentralized power and control [13-17].

\section{Actuators}

All types of the considered actuators have their advantages and disadvantages. Thus, different types of actuators are usually specialized and used for solving specific problems. In the case of large industrial works, where large capacities are required and massive loads are manipulated, pneumatic and hydraulic actuators are usually used, mainly due to greater economy compared to electric ones (which, however, does not negate the possibility of using the latter). As a consequence, large actuators, such as pneumatic and hydraulic actuators, which require external power supply or any other means of transmission, are not suitable when developing autonomous and miniature robotic systems.

For mobile robotic systems, servo drives are used most often because of their serial production (and therefore relatively low cost), reliability and ease of management. The main advantage of stepper drives is their accuracy. The supply of a control pulse to the drive winding causes the rotor to rotate by a strictly defined angle.

Stepper drive is used to automate individual units, where relatively high accuracy is required at a small angular velocity. However, there is a known problem associated with the possibility of "slipping" the rotor, which hinders the use of actuators based on stepper motors.

Actuators based on materials with shape memory and electroactive polymers have many advantages in the field of miniaturization and the repetition of the dynamics of real muscles and therefore have great potential. However, the high cost and the lack of an established industrial production makes the use of such actuators extremely difficult.

In the creation of a robotic module for performing swarm tasks several key aspects should be highlighted. 
Firstly, the task of creating the above-mentioned robot module assumes the creation of a module for the formation of three-dimensional structures with the contact connection of other modules, rather than distributed tasks, with considerable removal of the modules from each other. Consequently, the main purpose of the actuator in such a robotic module will be to activate some mechanism for connecting the modules and, possibly, a mechanism that would allow several modules to move in space.

Secondly, the task of modular robotics involves a compromise between the miniaturization of modules, their functionality and their cost. Proceeding from the analysis of the already implemented methods of combining homogeneous robots, the most preferable variant is a hybrid variant of connecting the modules with the help of mechanical and electromagnetic means. This is the most tested and reliable version of the connection with the highest speed of operation, allowing relatively fast connection and disconnection of modules, albeit with great mechanical complexity being a disadvantage.

Based on the analysis of different types of actuators, as well as various projects on modular robotics for the last 30 years, it can be concluded that the servo drive is best suited as an actuator for a mechanical connector. This type of actuator is smaller than a stepper motor and is capable of providing high accuracy in the control of the gripping mechanism, but the obvious disadvantage of this option is its relatively high cost [18]. Concerning the method of displacement in space, it is difficult to draw unambiguous conclusions, since the method of moving modules in space and, accordingly, the type of actuator for implementing the displacement depend on the design of the modules. An analysis of the existing solutions shows that at the moment the most efficient way of moving the modules is rotating a part of the module around the axis (Project Roombots) or using a two-motor movement mechanism of the MTRAN-III modules.

\section{Batteries}

The choice of type of batteries depends on their energy density, dimensions, cost and other parameters. Leadacid batteries have a rather long service life, are capable of giving off a large current, without a memory effect and have a very high operating temperature range. Leadacid batteries are widely used to power automotive systems, emergency power systems, and also for use as uninterruptible power supplies. However, this type of battery has such significant drawbacks in the form of large dimensions and mass, and a rather low specific energy density, which in the case of modular robotics is unacceptable.

Nickel-cadmium batteries were widely distributed in the world as power sources for portable electronics, but then significantly lost ground to nickel-metal hydride batteries. The latter can give relatively large currents and are best suited for powering devices with short and high current consumption. This type of battery is the only one that is recommended to be stored in a discharged state. It has such advantages as a very long service life, relatively short charging time and low cost; however, the disadvantages of these batteries (high self-discharge, relatively low energy density and "memory effect") make them unsuitable in the development of modern electrical devices.

Developed as a replacement for nickel-cadmium batteries, nickel-metal hydride batteries are successfully used in various fields. Having equal dimensions with nickel-cadmium batteries, nickel-metal hydride batteries have capacity three times higher than the former, but half the service life and high self-discharge (1.5-2 times). Also, nickel-metal hydride batteries are practically not influenced by the memory effect and require a full discharge before recharging only with long-term storage (more than 30 days). All these advantages in combination with stable output voltage make nickelmetal hydride batteries a good replacement for nickelcadmium as a power source for consumer electronics devices. In this case, high-capacity batteries (1500 $3000 \mathrm{mAh}$ ) are more suitable for devices that require high energy consumption for a short period of time (radio-controlled models, players, cameras, etc.). Batteries with a lower capacity (300 - $1000 \mathrm{mAh})$ are more suitable for cases when the energy consumption does not start immediately but after a considerable amount of time after charging or in the case of long-term or periodic energy consumption (walkie-talkies, hand lamps, GPS-modules, toys). Disadvantages of selfdischarge and service life are mostly eliminated in batteries with low self-discharge (LSD), but they have a lower capacity and a higher price. Such batteries are recommended for use in devices with high current consumption, as well as in devices with low consumption (watches, remote controls).

Lithium-cobalt batteries, being a traditional type of lithium-ion battery, surpass nickel-metal hydride batteries in a variety of parameters. They have low mass, high energy density, longer service life, low selfdischarge and they lack the "memory effect". These qualities allow lithium-cobalt batteries to find the widest application in electrotechnical devices. However, these batteries are subject to aging and loss of capacity even when not in use, which should be considered when designing the device.

Lithium-manganese batteries are safer than lithiumcobalt batteries and are capable of withstanding rapid charging by high currents, and also give current of up to $5 \mathrm{C}$. Because of this, they are most often used in devices with large current consumption: in high-power lanterns and radio-controlled models. The capacity of lithiummanganese batteries is slightly lower than that of lithium-cobalt batteries, and they are more sensitive to temperature changes; however, under operating conditions of lithium-manganese batteries (high current recoil), lithium-cobalt ones will most likely not be able to work and will break down because of overload.

Lithium-ferrophosphate batteries are very similar in characteristics to lithium-manganese batteries. The main differences lie in the lower cost and an ability to work under heavy loads (up to 10C). The structure of these batteries is such that, at critical loads, oxygen is not 
formed in the cell, which determines the increased safety of these elements. Unobtrusive, durable, resistant to low temperatures, lithium-ferrophosphate batteries are an excellent solution for powering devices operating under extreme conditions.

Lithium-polymer batteries, in fact, are an improved version of lithium-ion batteries. As an electrolyte, they use a polymer, which allows developers to create batteries of various shapes and sizes (including flexible ones). And although they are subject to aging and explosive during overloads, such advantages as low mass, high energy density, lack of "memory effect" and low self-discharge make this type of battery one of the best solutions for powering compact electrical devices. In the case of modular robotics, the size of the battery, its shape and mass play an extremely important role.

Based on the task of finding a balance between the optimal mass-scale parameters and the energy density, it is recommended to use lithium-polymer batteries for the development of homogeneous robotic modules. Of course, this choice imposes restrictions on the environmental conditions suitable for the operation of the device. Also, we should note new accumulators that have hydrogen, formic acid and other compounds.

\section{Communication}

When choosing the means of communication, one should consider the fact that in the case of rover robotics, the issue of the method of communication becomes even more significant, since the solution of the swarm tasks, both distributed and with direct contact interaction of the modules, requires a stable and fairly fast communication channel, both between the agents themselves and between the agents and the coordinator of the operation [19].

The communication problem is not trivial due to a number of physical limitations, the presence of a variety of interference, heterogeneity of the environment in which the modules are to work, etc. It should also be taken into account that there are different approaches to the algorithms of communication between agents. For example, the so-called tree topology does not imply a communication channel between all agents and is therefore fairly simple to use, while the network topology requires that each participant can take on the role of a communicator.

This organization is much more difficult to configure, but it provides much more fault tolerance, because failure of one of the network agents does not disrupt the functioning of the network as a whole. Because of this, the choice of the swarm control algorithm also influences the choice of the communication method. From a technical point of view, the chosen method and means of communication must correspond to the principles of compactness and economy, which are the main ones in modular robotics.

The considered methods of communication satisfy these parameters to varying degrees. The infrared channel (IR) is more robust to interference and external noise, safe, has an extremely low level of transmission errors, low power consumption and compactness, which is why it was used in the first mobile phones as a means of transferring media data for a relatively long time. Also, advanced code schemes for transferring data over the infrared channel provide a very high transmission rate. However, such disadvantages as the high cost of equipment (transmitters and receivers), as well as the need to maintain direct visibility between devices, make this method of communication not optimal for use in modular robotics.

The protocol that came to replace the infrared Bluetooth channel has significant advantages over IR technology. So, Bluetooth allows you to communicate at a relatively greater distance and create a connection with multiple devices at the same time, while also having a faster data transfer rate. In combination with low power consumption, these advantages generally make Bluetooth a good option for communication between modules.

However, it should be taken into account that Bluetooth operates under the "master-slave" principle and the interaction between master device and slave devices is possible, but between the slave device and several master devices is only realizable in more advanced devices with support for Bluetooth 4.1, the specification of which is somewhat ambiguous to meet the requirements of distributed networks. This imposes significant limitations on both the size of the swarm and the speed of the network.

The WiFi protocol has already become one of the main methods of wireless communication in contrast, it provides a greater number of simultaneously connected devices, as well as a longer connection range and a higher data transfer rate. For distributed networks, such characteristics are the best, but WiFi modules also have high power consumption, which is a critical disadvantage for the development of a compact robotic module. As a result, the use of WiFi technology is promising in modular robotics together with the development of batteries.

Finally, the radio modules working on the ZigBee protocol, specially developed for the creation of mesh networks, have a sufficiently long range for small dimensions (the range increases only with special antennas, which inevitably increases the overall dimensions), low power consumption, are capable of supporting a large number of modules, which is ideal suitable for a swarm network.

The disadvantages of this method of communication are a relatively low data transfer rate and low noise immunity, which, of course, is a significant problem in the work of modular robots in an extreme environment. However, the compactness, combined with the advantages already mentioned, makes this method of communication the most optimal for the development of modules, under the conditions of interacting at relatively short distances and without the need to work in an environment with a large amount of interference. It should also be taken into account that with the increase in the number of modular robots of up to a thousand or more, there is a serious problem of signal interference 
with the simultaneous exchange of data by all robots [20].

\section{Sensors}

The choice of sensor systems and sensors in homogeneous modular robots is, perhaps, one of the most difficult. In the creation of a modular robot there should be modules of a certain shape (cubic, spherical, parallelepipedal, etc.). In this regard, the sensors cannot be placed on the module body, since such arrangement of the sensors will complicate the dynamics of the modules and their interaction among themselves, and will also make the sensor itself vulnerable. As a result, the sensors used must be mounted in the housing, which, of course, will significantly increase the structural complexity of the module, most of which will be occupied by the propulsion unit and the battery. The use of sensors can also significantly increase the dimensions of the module, its mass and cost, since cheap sensors of standard sizes have rather large dimensions, and the reduction in the size of the sensors most often entails their substantial rise in price.

For these reasons, many projects to create homogeneous modular robots eliminated the use of sensors in modules, concentrating on their dynamics and the optimal method of connection. In this case, information about the environment of the module came from external sources (cameras surrounding the area of the robots, using the Kinect touch controller, etc.), or the modules did not have information about the environment and the location of other modules and were controlled directly by the operator. The ultimate goal is to create the most autonomous modules. However, on the basis of the comparative analysis of implemented modular robotic systems, it can be said that the higher the density of filling the module with functional elements (such as sensors), the more complex the module experiences when interacting with other modules.

The density of filling the module will also significantly affect the efficiency of the tasks. When performing tasks such as lifting the series-connected robots, quite large moments can act on the joints of the modules. As the practice of developers of already existing projects in modular robotics shows, the development of a complex self-sufficient selfreconfigurable modular robot results in a significant increase in the module size. For tasks involving holding modules, crawling and holding various objects, a large density will be a problem. Therefore, for problems of moving modules and other objects, an optimal choice between a complex design with a large functional and a small specific density requires a separate simulation. Due to the identified problems, further work on the project requires more precise definition of the working conditions of the robotic modules, a range of tasks that they can perform in view of the presented constraints, and, accordingly, a budget that can be incorporated into the creation of one module with specific parameters and functionality. Nevertheless, there are sensors, generally acceptable in size and price, for prototyping and clarifying emerging design difficulties, required modifications and missing information about the environment, which will require other internal or external sensors, or even a complete change in the design of the robotic module.

To implement the modular robot system, a MEMS gyro is recommended, which allows receiving information about the positioning of the module. Due to the cheaper production technology of MEMS systems, vibration MEMS gyroscopes have also become very cheap and widely available and often used in devices such as smartphones. Mass-dimensional parameters of such gyros are quite suitable for modular robotics. The question remains unclear as to what specific information about the external environment may be needed by the modules to perform specific tasks. However, in centering the modules relative to each other for connection, as well as determining the distance to the modules and obstacles around at the minimum range, an infrared distance sensor is miniature enough for insertion into the module frame. A sensor for determining the angle of rotation for the actuator is also required. In the case of a servo drive (recommended earlier for the development of mechanical connection of modules with hooks), a miniature turn sensor, based on the Hall effect (as in the dynamixel servo drives) and giving sufficient information about the angle of rotation of the motor rotor, is currently used. At the moment, the use of contact, sound and light sensors, as well as laser distance meters and GPS, is seen as inexpedient, since information from these devices at the moment cannot be classified as absolutely necessary for the operation of the modular robot.

\section{Conclusion}

The actuators, batteries, sensors and communication means, suitable for modular robotics, are considered. Further development of the robotic module systems and their design after the implementation of the declared functional will be possible after the testing of prototypes, and especially modeling and development of algorithmic support. To this end, at the next stage of the project, it is planned to analyze the limitations and justify the requirements for the model-algorithmic support for a single modular robot, which affect the methods of controlling pairwise connections of the ground-based robot. After this, it is planned to develop algorithms for controlling the connections of a swarm of terrestrial modular robots in the configuration of three-dimensional shapes.

This work is partially supported by the Russian Foundation for Basic Research (grant № 16-29-04101-ofi_m and grant № 1608-00696).

\section{References}

1. H. Ahmadzadeh, E. Masehian, Artificial Intelligence, 223, 27-64 (2015) 
2. P. Moubarak, P. Ben-Tzvi, Robotics and Autonomous Systems, 60, 1648-1663 (2012)

3. J. Baca, S.G.M. Hossain, P. Dasgupta, C.A. Nelson, A. Dutta, Robotics and Autonomous Systems, 62, 1002-1015 (2014)

4. M.S. Moses, H. Ma, K.C. Wolfe, G.S. Chirikjian, Robotics and Autonomous Systems, 62, 945-965 (2014)

5. J. Neubert, A. Rost, H. Lipson, IEEE Transactions on Robotics, 30(6), 1344-1357 (2014)

6. X. Wang, H. Jin, Y. Zhu, B. Chen, D. Bie, Y. Zhang, J. Zhao, Robotics and Computer-Integrated Manufacturing, 39, 56-67 (2016)

7. Kh. Taheri, H. Moradi, M. Asadpour, P. Parhami, Robotics and Autonomous Systems, 79, 72-86 (2016)

8. J. Baca, B. Woosley, P. Dasgupta, C.A. Nelson, Robotics and Autonomous Systems, 91, 284-298 (2017)

9. L. Bayındır, Neurocomputing, 172(8), 292-321 (2016)

10. J. Gonzalez-Gomez, H. Zhang, E. Boemo, Bioinspiration and Robotics: Walking and Climbing Robots, 403-428, (Maki K. Habib (Ed.), InTech, 2007)

11. N.E. Shlyakhov, I.V. Vatamaniuk, A.L. Ronzhin, IOP Conf. Series: Journal of Physics: Conf., 803, 012146 (2017)

12. N. Pavluk, A. Denisov, A. Kodyakov, A. Ronzhin, ICPEME 2016, MATEC Web of Conferences, 77, 04006 (2016)

13. A. Ivanov, O. Shmakov, SPIIRAS Proceedings, 49(6), 190-207 (2016)

14. A.I. Motienko, A.L. Ronzhin, N.A. Pavlyuk, Science Bulletin of the NSTU, 3(60), 147-165 (2015)

15. R.M. Yusupov, A.L. Ronzhin, From Smart Devices to Smart Space, Herald of the Russian Academy of Sciences, 80(1), 45-51 (2010)

16. A. Denisov, V. Budkov, D. Mikhalchenko, $I C R, 70-$ 79 (2016)

17. I. Vatamaniuk, G. Panina, A. Saveliev, A. Ronzhin, IEEE ICARSC, 306-310 (2016)

18. N. Pavluk, A. Ivin, V. Budkov, A. Kodyakov, A. Ronzhin, ICR, 113-123 (2016)

19. I. Vatamaniuk, D. Levonevskiy, A. Saveliev, A. Denisov, SPECOM, 588-595 (2016)

20. A. Saveliev, A. Ronzhin, Pattern Recognition and Image Analysis, 25(3), 517-525 (2015) 